\title{
PRESENTACIÓN
}

\section{Violencia de género, feminicidio y resistencias en tiempos pandémicos}

\section{Gender based violence, femicide and resistance in pandemic times}

Este número temático de la Revista O Público e o Privado titulado "Violencia de género, feminicidio y resistencias en tiempos pandémicos", concentra una serie de artículos que problematizan los distintos tipos y ámbitos de la violencia de género, sus formas más extremas como son los feminicidios, y las respuestas gubernamentales y resistencias sociales para prevenirlas, especialmente en el contexto de la pandemia por covid-19 que las exacerbó (PATERMAN ET AL., 2020). En efecto, la pandemia por el coronavirus agrava la "pandemia de violencia de género" considerada como tal en el año 2013 por la Organización Mundial de la Salud, ya que afecta al 35\% de población mundial, está presente en más de un continente y es de propagación comunitaria (oms, 2013). Por lo tanto, la violencia de género es uno de los mayores desafíos para la salud pública en Brasil, América Latina y el mundo, así como una de las violaciones a los derechos humanos más frecuentes y cotidianas, impidiendo una vida libre de violencias motivadas por el género (UNIFEM, 2003).

\section{La otra pandemia: violencia de género y feminicidios en una sociedad patriarcal-racista-neoliberal}

Según datos del informe "Violencia doméstica durante la pandemia de covid-19” elaborado por el Foro Brasileño de Seguridad Pública (FBSP, 2020), los casos de feminicidio crecieron 22,2\% entre marzo y abril de 2020 en 12 estados brasileños, en comparación con los datos de 2019. El mismo informe apunta a una reducción en el número de casos de lesiones corporales intencionales - cuando hay intención de cometer agresión - del 25,5\%. Tal reducción está directamente relacionada con la dificultad de acceder a las instituciones policiales en el período y, por lo tanto, de hacer la denuncia correspondiente. 
$\mathrm{Si}$, por un lado, los registros de casos han disminuido por las razones presentadas, el proceso de subregistro es revelador cuando, por otro lado, se observa un incremento del 17,9\% de las denuncias realizadas por teléfono, en comparación entre los meses de marzo de 2019 a 2020. En el mes de abril, cuando comenzó la cuarentena en la mayoría de los estados brasileños, el aumento fue del 37,6\% con respecto al año anterior. En general, los datos indican un aumento en la exposición de las mujeres a situaciones de violencia directa debido a la imposición de compartir más tiempo con los compañeros y a la dificultad de buscar apoyo durante el período (BARRoso, 2020).

Para Barroso (2020), la necesidad de aislamiento físico o distanciamiento y la fragilidad (y ausencia, en la mayoría de los casos) de los servicios de protección social para las mujeres explican estos datos. En el primer caso, la convivencia más cercana y permanente con los agresores facilitaría tanto las situaciones de tensión más recurrentes -la acumulación de actividades con nuevos requerimientos de aseo e higiene, el cuidado a tiempo completo de niños, niñas y personas mayores, entre otros- así como también un obstáculo directo para buscar una comisaría u otras instituciones de servicios especializados. Además, a pesar del aumento de registros, es necesario considerar la brecha entre los datos y la realidad vivida por las mujeres, hecho que se despliega en un escenario de subregistro significativo.

Más allá de este subregistro, se observa un predominio de los análisis en el ámbito doméstico y en las relaciones afectivo-maritales, que terminan invisibilizando otros contextos y expresiones de violencia de género, como el trabajo doméstico (remunerado y no remunerado), la violencia LGBT-fóbica o la violencia contra mujeres racializadas, con énfasis en el genocidio de pueblos indígenas, que afecta particularmente y tiene diferentes implicaciones para los cuerpos-territorios de las mujeres indígenas (GAGo, 2020). Así, paralelamente al subregistro y a la brutalidad con la que se da la violencia contra las mujeres, existe una sofisticada combinación de viejas y nuevas expresiones de violencia de género que no se restringen al contexto de la pandemia covid-19, sino que son producto y productoras de la sociedad patriarcal-racista-capitalista (DAVIs, 2017; SAFIOTTI; 2004), que se profundizan en su versión neoliberal, 
contribuyendo muchas veces a la aprehensión fragmentada del fenómeno y, en consecuencia, a su naturalización.

En efecto, en Brasil, la encuesta mensual realizada por el Centro para el Estudio de la Violencia de la usp y el Foro Brasileño de Seguridad Pública apunta que los primeros seis meses de 2020 vieron un aumento en el número de mujeres víctimas de violencia doméstica en Brasil, en comparación con el mismo período del año pasado. Según un estudio del 'Monitor de la Violencia', las principales víctimas del feminicidio son las mujeres negras. En los primeros seis meses de 2020,1.890 mujeres fueron asesinadas de manera violenta, gran parte de ellas en medio de la nueva pandemia de coronavirus, un aumento del $2 \%$ con respecto al mismo período en 2019. Según el estudio, 631 de estos delitos fueron motivados por el odio por la condición de género, es decir, feminicidio. La violencia intrafamiliar también afecta más a las mujeres negras, representando el 58\% de las llamadas al número de asistencia 180. Asimismo, son las más afectadas por la mortalidad materna (56\%), y la violencia obstétrica (65\%). Por todo esto, el reciente seminario "Mujeres negras mueven Brasil: visibilidad y oportunidad”, promovido por la Cámara Federal de Diputados en el Día de la Conciencia Negra (AgEncia CÁmara, 2020) concluyó que las mujeres negras en Brasil son el segmento de la población donde se concentra el mayor número de feminicidios, además de ser las que más sufren por violencia doméstica y obstétrica, mortalidad materna y por la criminalización del aborto.

Por lo tanto, en el actual contexto de la pandemia del covid-19, ante los crecientes niveles de violencia de género (PATERMAN ET AL., 2020), se implementaron distintas estrategias públicas y se activaron redes territoriales de solidaridad y de denuncia en contextos complejos de recesión económica y confinamiento social ${ }^{1}$, aunque los resultados han sido escasos. Peor aún, en algunos países los estados siguieron contribuyendo a su impunidad y agravamiento, al no promover su prevención, investigación, sanción y reparación desde estrategias integrales (LAGARDE, 2006; SAGOT, 2020).

1. Ver por ejemplo en Argentina las medidas en materia de género y diversidad en el marco de la emergencia sanitaria (Ministerio de las Mujeres, Géneros y Diversidad, 2020): https://www.argentina. gob.ar/sites/default/files/medidas_durante_la_emergencia_sanitaria_mmgyd.pdf 


\section{Los avances y las dificultades en la lucha por erradicar la violencia de género y los feminicidios a nivel internacional y en la región}

Estas dificultades para reducir los niveles de violencia contra las mujeres responden a sus raíces históricas en la desigualdad estructural de género y en la "interseccionalidad" de las discriminaciones, tal como lo advierte la Recomendación General N² 28 del Comité CEDAw: "la discriminación de la mujer por motivos de sexo y género está unida de manera indivisible a otros factores que afectan a la mujer, como la raza, el origen étnico, la religión o las creencias, la salud, el estatus, la edad, la clase, la casta, la orientación sexual y la identidad de género." Además, responde al diseño estatal moderno -y por lo tanto a sus capacidades- que históricamente separaron el ámbito público del privado, privilegiando la seguridad en el espacio público, dejando desprotegido el ámbito privado (MAFFíA, 2020).

Sin embargo, desde la proclamación de que "lo personal es político" y "hacer de lo privado, público", el feminismo logró impactar en el sistema internacional, apelando a la intervención del Estado y por lo tanto, de las Naciones Unidas, en la esfera doméstica (DíEz PERALTA, 2011; GuSTAVSEN ANGELINI, 2018; HALl, 2015). En la Declaración sobre la Eliminación de la Violencia Contra la Mujer (Resolución 48/104), aprobado en 1993, se identificaron los tipos de violencias contra la mujer y los ámbitos en los que suelen ocurrir como en el seno familiar, en la comunidad y los ejercidos o tolerados por el Estado. Asimismo, se señalaron las responsabilidades del Estado en la materia, tanto para prevenir como para investigar y sancionar cualquier acto de violencia contra la mujer. En esta misma línea, a nivel regional, se destaca la Convención Interamericana para Prevenir, Sancionar y Erradicar Cualquier Forma de Violencia Contra la Mujer (convención de belem do PaRÁ, 1994).

Para que fuera posible la incorporación de la violencia contra las mujeres en la agenda internacional, el movimiento feminista utilizó de forma estratégica la "universalidad de los derechos humanos" para combatir la impunidad estatal en la materia (DíEz PERALTA, 2011; HALl, 2015). Así logró mostrar cómo la violencia de género afecta la vida cotidiana de las mujeres impidiendo que vivan una vida libre de violencia, lo cual es un derecho humano fundamental que debe ser garantizado por el Estado, pero al mismo tiempo, planteando 
las limitaciones estatales para reducirlas, llevándolo a la arena internacional (DUNNe et AL., 2013; GUSTAVSEN ANGelini, 2018; Unifem, 2003).

Por lo tanto, el movimiento feminista se planteó inicialmente una mirada transnacional y más recientemente, glocal. En efecto, ante el universalismo de los derechos humanos, se objetó que no se daba cuenta de las identidades locales y de las experiencias atravesadas por la edad, la religión, clase y etnia racial, ya que se basaba en las problemáticas de las mujeres de los países más ricos que permitía una lectura regresiva en términos de demanda de protección (feminismo del 1\%), donde las mujeres del Sur global aparecían siempre como víctimas perpetuas de su cultura, justificando la intervención y ocupación (FRASER, 2008; GUSTAVSEN ANGELINI, 2018; halL, 2015). Ante esto se postuló la visión glocal, que incluye la demanda por democratizar los espacios de activismo a nivel internacional y la necesidad de plantear estrategias de largo plazo que no sean sólo penales, sino que también promuevan el cuidado y el bienestar en la comunidad. Desde esta visión crítica, si bien se reconocen los avances ocurridos anteriormente, se advierte que parten de una visión individualizada y despolitizada de la violencia contra las mujeres, planteando soluciones policiales y jurídicas punitivas que se focalizan en el individuo y no en el cambio social estructural.

Así, desde las posturas críticas feministas, se señala que el sistema internacional está definido por jerarquías de género que refuerzan su subordinación y que por lo tanto, en la medida en que no se cuestionen las estructuras globales de opresión y de desigualdad económica y social (racismo, colonialismo y capitalismo), no se podrá erradicar la violencia contra la mujer de manera colectiva y permanente (MERRY, 2006 CITADA EN GUSTAVSEN ANGELINI, 2018; KAPUR, 2002, CitAda en Hall, 2015). Por lo tanto, en la actualidad, las demandas de parte del movimiento feminista incluyen no sólo la provisión de asistencia y el acceso a la justicia para las víctimas individuales, sino también la transformación de las estructuras de poder que reproducen las condiciones que perpetúan la violencia hacia las mujeres. Del mismo modo, aunque se invoca al Estado como responsable de la prevención, investigación y sanción de la violencia contra las mujeres, se advierte que el Estado también reproduce y legitima las estructuras de desigualdad y la violación de los derechos humanos (sAGOT, 2020). 
Estas mismas tensiones se encuentran comprendidas en el recorrido teórico-político del concepto de femicidio, acuñado por primera vez por Diana Russell y Jill Radford en 1992. Más allá de la definición elaborada en la Cuarta Reunión del Comité de Expertas/os (cEvI), celebrada el 15 de agosto de 2008², la antropóloga Rita Segato (2003) comprende al femicidio como la muerte de una mujer producida por un hombre debido a su condición de género, que implica la apropiación del cuerpo de la mujer por el hecho de serlo o no serlo de manera "adecuada", como castigo por romper la dependencia y, por tanto, la dominación masculina y como modo de convertirla en "víctima sacrificial", como prueba para participar de la economía simbólica de los pares, como parte de la denominada "masculinidad hegemónica" (CONNELl y MEsSERSCHMidT, 2005; OtAMENDi, 2020; PAGe, 2009; stroud, 2012). Así, el cuerpo de la mujer se convierte en un objeto propiedad del agresor, quien, a partir de celos, infidelidad o fin de una relación, toma para sí lo que creía poseer (HERNÁNDEZ ET AL., 2018). Es la culminación de estructuras de desigualdad, subordinación y poder que termina con la vida objeto de deseo.

Luego, la visión crítica del rol estatal en la violencia de género se ve reforzada en el concepto de feminicidio acuñado por la feminista mexicana Marcela Lagarde para enfatizar que es una violencia ejercida por "hombres colocados en supremacía social, sexual, jurídica, económica, política, ideológica y de todo tipo, sobre mujeres en condiciones de desigualdad, de subordinación, de explotación o de opresión, y con la particularidad de la exclusión" (LAGARDE, 2006:221) y que se ejerce violencia institucional que conduce a la impunidad cuando existe "discriminación en la impartición de justicia, la discriminación en las averiguaciones, en los peritajes" (LAGARDE, 2006:223). Por su parte, las autoras Rosa Linda Fregoso y Cynthia Bejarano (2010: 5) también reformulan el concepto para poner en cuestión las relaciones de poder que le dan sustento: a partir de la conceptualización de Lagarde, definen feminicidio como el "asesinato de mujeres y niñas basados en una estructura de poder de género".

Por lo tanto, como ya se señaló, para prevenir la violencia de género y los feminicidios, se promueven las estrategias glocales, ya que tienen en cuenta el

2. https://www.oas.org/es/mesecvi/docs/DeclaracionFemicidio-ES.pdf 
nivel de lo local, pero también el estatal y transnacional, buscando articular y superar las limitaciones de cada nivel, no perdiendo de vista el territorio, y las luchas y resistencias de los movimientos feministas, pero tampoco la incidencia de las diferentes agencias estatales e internacionales, poniendo en cuestión las estructuras de poder desiguales y promoviendo masculinidades alternativas, y los derechos, las libertades y la autonomía de las mujeres y diversidades.

En este sentido, en las últimas cuatro décadas, las prácticas de violencia en las relaciones privadas de la familia, especialmente contra niños y mujeres, se han ido constituyendo progresivamente como un problema social. Comúnmente llamada violencia doméstica, la violencia contra la mujer en el ámbito familiar es un tema prioritario en las agendas políticas internacionales y nacionales. En 2011, el Parlamento Europeo define la Política Marco para Combatir la Violencia contra las Mujeres y el Consejo de Europa establece el Convenio para la Prevención y Combate de la Violencia contra las Mujeres y la Violencia Doméstica, un documento ratificado por Portugal en 2013 y vigente desde el 1 de agosto de 2014. Internamente, varios países europeos implementaron planes nacionales para combatir la violencia y la discriminación contra la mujer. Por su parte, la ley española (LEY ORGÁNICA 1/2004) establece medidas integrales de protección contra la violencia de género, incluyendo mecanismos de formación humana con inserción de asignaturas desde la primaria hasta la universitaria donde se busca promover el respeto de los derechos, las libertades y la igualdad entre hombres y mujeres, así como la tolerancia al ejercicio y la libertad dentro de los principios democráticos de convivencia. De esta manera, el sistema educativo español busca eliminar las barreras a la plena igualdad entre hombres y mujeres, y la formación para la prevención y resolución pacífica de conflictos.

En cuanto a la región de América Latina y el Caribe es posiblemente la que más ha avanzado en la creación de marcos regulatorios nacionales para abordar la violencia contra la mujer. En "violencia contra las mujeres en América Latina”, Natalia Ghirardi (2016), hace consideraciones sobre el acceso a la justicia y los factores estructurales que multiplican los femicidios. Señala que en una primera etapa, la región se enfocó en normas dirigidas a la violencia en las relaciones familiares o violencia doméstica. En ese sentido, cabe 
destacar la Ley 11.340, de 08/07/2006 (LEY MARIA DA PENHA) que es considerada por las Naciones Unidas (oNU), la tercera mejor ley del mundo en la lucha contra la violencia doméstica, solo superada por España y Chile. Luego, en la última década, una docena de países se han movido hacia la aprobación de leyes de protección integral contra diversas formas de violencia de género, orientadas a la violencia que ocurre no solo entre miembros de la familia o unidades domésticas, sino también en los diferentes entornos de la comunidad. De esta forma, países como Argentina, Bolivia, Colombia, El Salvador, Guatemala, México, Nicaragua, Panamá, Perú y Venezuela sancionaron leyes de segunda generación, incorporando la definición de otras formas violencia de género institucional, laboral, obstétrica, mediática, contra derechos reproductivos, acoso sexual, violencia patrimonial y simbólica.

Sin embargo, a pesar de los recientes avances en la legislación, estudios e investigaciones sobre el tema han señalado el aumento y la gravedad de esta violencia en las últimas décadas mostrando también su carácter estructural (LOINAZ ET AL., 2018; LÓPEZ PONs, 2010), pero también una mayor visibilidad como resultante de los movimientos de resistencia como \#NiUnaMenos en Argentina y el colectivo feminista chileno "Las Tesis", entre otros (sciortino, 2018).

\section{Esta convocatoria: violencias motivadas por el género, respuestas estatales y resistencias en tiempos pandémicos}

En el contexto de la cuarentena a raíz de la pandemia de Covid-19 muchas víctimas deben convivir con sus agresores, limitando el acceso a redes locales de solidaridad y a las capacidades estatales más orientadas a la seguridad en el espacio público que a la protección de los hogares. Este panorama obliga, cada vez más, a repensar en la dinámica y las diversas expresiones de la violencia contra las mujeres, así como en las estrategias de prevención y resistencias para revertir este proceso y la violencia simbólica que la respalda.

En este sentido, este número temático de la Revista recorre, en el primer eje, distintos tipos y modalidades de la violencia de género; en el segundo, algunas políticas públicas al respecto, y en el tercero, las estrategias de resistencia, siendo estos ejes atravesados en algunos casos por los desafíos actuales durante la pandemia del covid-19. En cuanto al primer eje, los artículos analizan cómo 
la violencia de género en la vida cotidiana de las mujeres, ya sea transitando por las calles en un barrio popular como participando en el mundo digital, se vio exacerbada en el contexto de la pandemia.

En cuanto al segundo eje, los artículos evalúan cómo, ante este agravamiento durante la crisis sanitaria, se generaron diferentes dispositivos estatales, desde campañas publicitarias, mayor atención telefónica hasta nuevas disposiciones normativas. Se observan también las dificultades en la articulación de los niveles estaduales con el federal. Además, se advierte que la mayoría de las respuestas estatales siguen basándose en una lógica punitiva donde el énfasis está puesto en la denuncia contra el agresor, pero no en fortalecer la autonomía, las libertades y los derechos de las mujeres.

Por último, los artículos del tercer eje tratan distintas estrategias de resistencias feministas, como el movimiento en las calles por el feminicidio y el aborto, o como el \#MeToo en el cine, permitiendo visibilizar la violencia de género y la división sexual del trabajo, así como también las distancias que existen entre las demandas y representaciones en las calles, y los discursos que circulan en el ámbito legislativo.

\section{Eje temático: violencia de género en el espacio urbano y en el digital.}

Tal como se indicó, en el primer eje se presentan distintas modalidades y tipos de la violencia motivada por el género. . En primer lugar, el artículo de Paz Cabral titulado "Ser mujer joven en Melchor Romero Prácticas espaciales, movilidades y violencias en perspectiva de género", analiza, a partir de un trabajo etnográfico, las violencias ejercidas contra mujeres jóvenes en el espacio público en un barrio popular. Estas experiencias, sus temores, y los controles ejercidos por sus responsables y los juicios morales que pesan sobre ellas, llevan a que las jóvenes permanezcan menos tiempo en la calle y más tiempo en sus casas, reactualizando la tradicional división de género entre el espacio doméstico y el espacio público. Así, las jóvenes ven limitada su autonomía en el uso del espacio, del tiempo y de sus cuerpos en el territorio. Para prevenir sufrir robos, violencias sexuales y acoso callejero, restringen sus libertades y deseos, internalizando estigmas y miedos, y reproduciendo el estereotipo de las mujeres como vulnerables. Sin embargo, también hay resistencias y desafíos: la agencia 
de estas jóvenes las lleva a disputar esos sentidos y a "vivir sus corporalidades, practicar sus deseos, ejercer sus sexualidades" aún con las limitaciones impuestas ante la interseccionalidad de ser mujeres, jóvenes y de sectores populares.

Así como en el artículo anterior se observa la violencia de género en el espacio urbano, en el artículo de Karina Nohemí Martínez Meza y Arturo Miguel Chípuli Castillo titulado "Violencia en espacios digitales en México. Una mirada desde la pandemia de Covid-19" se muestra cómo en el espacio digital también se dan prácticas de violencia de género. En efecto, allí se reproducen estereotipos y desigualdades de género, y discriminación hacia la mujer, afectando su "reputación digital"y por lo tanto, generando daños psicológicos, sexuales, e incluso patrimoniales y físicos. Para ello, se describen los tipos de violencia, sus modalidades y las conductas que producen violencia de género en espacios virtuales. Teniendo en cuenta lo anterior, se citan los datos de México sobre ciberacoso, donde predomina el tipo de violencia sexual que se ve facilitada por el desconocimiento de la identidad del agresor vinculada con su anonimato. Es esperable que tales tendencias también se hayan incrementado durante la pandemia de covid-19, más aún por el aumento de la participación en el espacio digital debido al confinamiento. En efecto, los cibernautas encuentran en dicho espacio nuevas formas de socialización, incluyendo nuevos canales para la agresión y la violencia de género. Ante esto, se demanda que el estado adopte un enfoque de género en las políticas públicas que regulan el espacio virtual, incluyendo la tipificación de delitos informáticos vinculados a la violencia de género y programas eficaces para prevenirlas y erradicarlas.

\section{Eje temático: respuestas estatales ante la violencia de género y sus limitaciones en tiempos pandémicos}

En cuanto a las respuestas estatales ante la violencia de género, especialmente durante la crisis sanitaria, se observan ciertas limitaciones. Por un lado, el texto de Anderson Oliveira, Juliana Tonche y Mariana Possas, titulado "Victimización de la mujer y covid-19: entre la permanencia y el agravamiento", parte de la comprensión del agravamiento de la violencia doméstica contra las mujeres en el contexto de la pandemia y presenta una reflexión sobre las medidas institucionales para abordar el problema. Como crítica, las/ 
os autores señalan un predominio de la lógica punitivista como principal respuesta a la violencia intrafamiliar contra la mujer, lo que ha derivado en una débil protección de la mujer en estos casos.

Por su parte, el texto "Políticas públicas sobre violencia intrafamiliar en tiempos de la pandemia covid-19: acciones de los órganos de política estatal para las mujeres en Brasil", de Paola Stuker, Krislane de Andrade Matias y Joana Luiza Oliveira Alencar, también observa un aumento de la violencia contra las mujeres en el contexto de la pandemia del covid-19. Enfocado en el análisis de las acciones realizadas por los Organismos de Políticas de la Mujer durante la pandemia en Brasil, señala la generación de iniciativas más rápidas y comprometidas en este período en los estados que cuentan con dichos entes en comparación con los que no tienen dicha estructura. Aún así presentan limitaciones en sus respuestas que pueden deberse a la desestructuración de las políticas públicas para las mujeres a nivel federal.

\section{Eje temático: resistencias feministas, de la calle a la cultura y las artes}

En cuanto a las resistencias, en "De las calles al parlamento: los efectos de las agendas feministas en la esfera política”, de Anelise Gregis Estivalet y Gabriel Dvoskin, se aborda una nueva forma prometedora de estado feminista, transnacional, de múltiples escalas y posbienestar que debe marcar acciones de la nueva ola feminista. En este trabajo, sus autores proponen analizar las repercusiones obtenidas en el ámbito político, tanto en Argentina como en Brasil, a partir de los signos que dieron lugar a movimientos feministas como “Ni Una Menos”. Así se preguntan si las representaciones y discursos puestos en circulación a nivel social fueron retomados en proyectos legislativos presentados dentro de las temáticas de género y sexualidad, durante el período de estudio, a partir del análisis de los debates sobre la legalización del aborto y en torno al feminicidio. Allí observaron que se mantiene una distancia significativa entre las representaciones que circulan sobre estos temas a nivel social y las producidas a nivel legislativo.

Por su parte, Mirla Cisne, Luana Gomes Medeiros y Viviane Vaz Castro en "Detrás de la pantalla de cine, hay violencia contra las mujeres: un análisis de la importancia del movimiento \#MeToo", buscan analizar, desde una 
perspectiva feminista materialista, la violencia contra las mujeres en el cine a través de tres casos denunciados por el Movimiento \#MeToo, contra los directores Bernardo Bertolucci, Woody Allen y el productor Harvey Weinsten. Además del análisis de las denuncias, destacan la importancia del Movimiento \#MeToo, así como la forma en que las mujeres comenzaron a organizarse contra la violencia en el cine. La problematización del patriarcado y la división sexual en el cine es una base para el análisis del objeto propuesto.

\section{Alejandra Otamendi ${ }^{1}$ Milena Fernandes Barroso ${ }^{2}$ Márcia Esteves de Calazans ${ }^{3}$}

Organizadoras

1. Doctora en Sociología en cotutela por la Universidad de Buenos Aires (UBA) y eness de Paris. Magister en Gobernanza Global y Diplomacia, Universidad de Oxford. Licenciada en Sociología, ubA. Investigadora del Instituto de Investigaciones Gino Germani de la uвA. Co-coordinadora regional del Grupo de Trabajo de CLACso "Violencias, políticas de seguridad y resistencias". http://orcid.org/0000-0002-8271-7632 otamendialejandra@gmail.com

2. Doctora en Trabajo Social por la Universidad Estatal de Río de Janeiro (UERJ), con una pasantía de doctorado en la Universidad de Québec, Canadá. Profesor del Programa de Posgrado en Trabajo Social y Sustentabilidad y del curso de Trabajo Social de la Universidad Federal del Amazonas (UfAm). https://orcid.org/0000-0002-8349-1508 mibarroso@yahoo.com.br

3. Doctora en Sociología por la Universidad Federal de Rio Grande do SulUfRgs y postdoctorada por el Instituto Nacional de Ciencia y Tecnología INCT/ CNPQ: Violencia, Democracia y Seguridad Ciudadana, Universidad de São Paulo (usP). Actualmente se encuentra realizando una Pasantía Postdoctoral en el Programa de Posgrado de la Facultad de Educación de la Ufrgs, junto con el grupo de investigación PEABIRU: Educación e Interculturalidad Amerindia (pnpd/capes). Miembro del Grupo de Trabajo de clacso "Políticas de violencia, seguridad y resistencia"

https://orcid.org/0000-0002-8591-1828 marcia_calazans@hotmail.com 


\section{Referencias}

BARROSO, M. F. Claves para analizar la violencia contra las mujeres en tiempos de crisis: enfoques y desafíos en el contexto de la pandemia en Brasil. Revista Vértices. Dossier Temático "Violencia de Estado y política social: entre el aparato asistencial y la criminalización de la cuestión social". v.22, 2020.

CRENSHAW, Kimberlé W. Demarginalizing the intersection of race and sex: a black feminist critique of discrimination doctrine, feminist theory and antiracist politics. University of Chicago Legal Forum, 1989.

CONNELL, R. W. y J. W. MESSERSCHMIDT. Hegemonic masculinity: Rethinking the concept. Gender \& Society, 19, 2005, p. 829-859.

DAVIS, A. Mulheres, cultura e política. São Paulo: Boitempo, 2017.

DÍEZ PERALTA, E. Los derechos de la mujer en el derecho internacional. Revista Española de Derecho Internacional. LXIII/2: 2011, 87-121.

DUNNE, T., KURKI, M., \& SMITH, S. International Relations Theories (Third ed.). Oxford: Oxford University Press, 2013.

Fórum Brasileiro de Segurança Pública (FBSP). Nota técnica: violencia doméstica durante la pandemia de covid-19. 16 abr. 2020. Disponível em: https:// forumseguranca.org.br/wp-content/uploads/2018/05/violencia-domestica-covid19-v3.pdf. Acesso em: 30 ago. 2020.

FRASER, N. Unruly practises: power discourse and gender in contemporary social theory. Minnesota: University of Minnesota Press, 1989.

FREGOSO, R., \& BEJARANO, C. Introduction: A cartography of feminicide in the Americas. En R. Fregoso \& C. Bejarano (eds.), Terrorizing women: Feminicide in the Americas (pp. 1-42). Durham, NC: Duke University Press, 2010.

GAGO, V. A potência feminista, ou o desejo de transformar tudo. São Paulo: Elefante, 2020.

GHERARDI, N. Violência contra mulheres: Considerações sobre acesso a justiça e as condições estruturais em que os feminicdios se mulltiplicam. Revista SUR 24 - v.13 n.24. p, 129-136, 2016. 
GUSTAVSEN ANGELINI, G. Gender violence in Argentina: the role of women in support and prevention mechanisms from civil society to gender policy-making. Tesis de maestría. Department of International Environment and Development Studies. Noragric, 2018. URL: https://brage.bibsys.no/xmlui/bitstream/ handle/11250/2502980/Angelini2018.pdf?sequence=1\&isAllowed=y

HALL, R. J. Feminist Strategies to End Violence Against Women. En Rawwida BAKSH, R. y HARCOURT, W. (eds.) The Oxford Handbook of Transnational Feminist Movements. Oxford: Oxford University Press, 2015. URL: http://www.oxfordhandbooks.com/ view/10.1093/oxfordhb/9780199943494.001.0001/oxfordhb-9780199943494 HERNÁNDEZ, W.; M. RAGUZ; H. MORALES y A. BURGA. Feminicidio: determinantes y evaluación del riesgo. Lima: Universidad de Lima, Instituto de Investigación Científica, 2018. URL: http://repositorio.ulima.edu.pe/bitstream/handle/ ulima/5025/Hernandez_Raguz_Morales_Burga.pdf?sequence=3\&isAllowed=y LAGARDE, M. Del femicidio al feminicidio. Desde el jardín de Freud: Revista de Psicoanálisis, 6, 216-225, 2006.

LOINAZ, I., MARZABAL, I., Y ANDRÉS-PUEYO, A. Risk factors of female intimate partner and non-intimate partner homicides. The European Journal of Psychology Applied to Legal Context, 10, p.49-55, 2018.

LÓPEZ PONS, M. M. La violencia de género en el territorio latinoamericano, a través de la ocurrencia creciente de los feminicidios en la región. Revista Latinoamericana de Geografia e Gênero, 1(1), p.78-88, 2010.

MAFFÍA, D. Violencia de Género: ¿La otra pandemia?, Grimson, A. (dir.) El futuro después del COVID-19. Buenos Aires: Jefatura de Gabinete de Ministros, 2020. URL: https://www. argentina.gob.ar/sites/default/files/el_futuro_despues_del_covid-19_0.pdf

MINISTERIO DE LAS MUJERES, GÉNEROS Y DIVERSIDAD. Medidas en el marco de la emergencia sanitaria. Buenos Aires: MMGyD, 2020. URL: https://www.argentina. gob.ar/sites/default/files/medidasanteemergenciasanitaria_2.pdf

ORGANIZACIÓN MUNDIAL DE LA SALUD. Global and regional estimates of violence against women: prevalence and health effects of intimate partner violence and non-partner sexual violence. Ginebra: OMS, 2013. URL: http://www.who.int/ reproductivehealth/publications/violence/9789241564625/en/ 
OTAMENDI, M. A. Suicidios, femicidios-suicidios y armas de fuego en Argentina. La masculinidad hegemónica en debate, Revista Ciencias Sociales, Universidad de la República, 33, 46: 107-130, 2020. URL: https://rcs.cienciassociales.edu.uy/index. $\mathrm{php/rcs/article/view/39/29}$

PAGE, E. Men, masculinity and guns: Can we break the link? Londres: IANSA, 2009. PETERMAN, POTTS, O'DONNELL, THOMPSON, SHAH, OERTELT-PRIGIONE, Y VAN GELDER. Pandemics and Violence Against Women and Children, CGD Working Paper 528. Washington, DC: Center for Global Development, 2020. URL: https://www. cgdev.org/publication/ pandemics-and-violence-against-women-and-children RADFORD, J. Y RUSSELL, D. Femicide: The Politics of Woman Killing. Nueva York: Editorial Twayne, 1992.

SAFFIOTI, Heleieth. Género, patriarcado, violencia. São Paulo: Perseu Abramo, 2004. SAGOT, M. Violence Against Women: Contributions from Latin America. En Bada, X., Rivera-Sánchez, L. The Oxford Handbook of the Sociology of Latin America. Oxford: Oxford University Press, 2020. URL: https://www.oxfordhandbooks.com/ view/10.1093/oxfordhb/9780190926557.001.0001/oxfordhb-9780190926557-e-32 SEGATO, R. Las estructuras elementales de la violencia. Buenos Aires: Editorial Prometeo UNQUI, 2003. URL: https://drive.google.com/ file/d/0Bzi8hpy848BJRjVnTWNuNFhUa3c/view?usp=sharing

SCIORTINO, S. Consideraciones sobre el movimiento amplio de mujeres a partir del "Ni Una Menos": continuidad histórica, diversidad y trayectorias locales. PUBLICAR-En Antropología y Ciencias Sociales, Argentina, 0, jul. 2018. URL: http:// ppct.caicyt.gov.ar/index.php/publicar/article/view/13338 .

STROUD, A. Good guys with guns: Hegemonic masculinity and concealed handguns. Gender \& Society, 26(2), 216-238, 2012.

UNIFEM (2003). Not a minute more. Ending Violence against Women. Nueva York, United Nations Development Fund for Women (UNIFEM), 2003. URL: https://www. coe.int/t/dg2/equality/domesticviolencecampaign/Source/PDF_UNIFEM_No_a_ minute_more_2003.pdf 